\title{
How a Two-Dimensional Approach of the Physical Time Can Describe That the Net Temporal Movement of the Universe is a Constant Zero. Why We Do Not Meet With Our Anti-Selves
}

\author{
Prasenjit Debnath \\ PhD Student, NIT Agartala, India
}

\begin{abstract}
A two-dimensional approach of the physical time is nothing but the expression of time in terms of real time component along the $x$-axis and imaginary time component along the y-axis. As we know, the space and the physical time are interdependent on each other; it is not unwise to think that every space point is the elemental representation of Universe's space and every space point is associated with a unit vector of time with respect to the reference frame. Matter and energy are nothing but the space deformations. We can use the term virtual particles to represent any kind of energy. Actually, matter and energy are two opposite facets of the same coin. Real particles are responsible for matters while virtual particles are responsible for energies. Any particle, does not matter it is real or virtual particle, is actually deformation of space. In other words, asymmetric distributions of space points are responsible to produce different matters and different energies. The physical time runs at different speeds at different deformations of space points. The speed of the physical time is proportional to the degree of deformation of space points which form a local area of symmetry (any particular local area has only even distribution of space points within that local area, as a result, the resultant macroscopic time is symmetric and identical for every space points within that local area which means that the resultant macroscopic representation of time along $x$-axis, which can be perceived by human race, is same at every space point within the local area but asymmetric between different local areas which means every local area has a unique resultant macroscopic time). The unit vector of time of solid has different direction compared to the unit vector of time of liquid or the unit vector of time of gas. As, real time (the x-axis component of the physical time) runs slower for solid compared to liquid and gas, the $x$-axis component of the unit vector of time is lower compared to $x$-axis component of liquid and gas. In other words, the unit vectors of time of solid are more vertically oriented compared to the horizontal component. As the real time (time along $x$-axis) can be recognizable by human race, time runs slower in solid compared to liquid and gas of the same amount. The magnitude of unit time vector is always the same but it varies only in direction in the first quadrant for ordinary matters. But the macroscopic time is always additive for ordinary matters, does not matter whether it is in solid or liquid or gaseous form. Also the macroscopic time is additive for positive energy. But the macroscopic time is subtractive for anti-matters and negative energies. It implies that because in our Universe has very little anti-matters which is not substantial to reduce the speed of time to the present state, there must be huge amount of negative energy available in the Universe to reduce the speed of time to the present state which is acceptable by living being to synchronize themselves with. We know that gravity is a negative energy because we need to do positive work against it to get rid of it. There must be enormous debt of positive energy with respect to the negative energy; the positive energy is in chase to catch the negative energy to reduce the debt, and in this process, the positive energy keeps on forming the ordinary matters to reduce the debt and when it forms the ordinary matter, the matter gives rise of gravity of itself which is again making the debt the same or more but not less. The dominant negative energy is reducing the speed of time to the present abstract rate of time in the Universe. The parallel Universes tell us that every matter has an anti-matter. The energy has its anti-energy, if energy is positive and attractive; the anti-energy is negative and repulsive. The same is true for negative energy too. Then why do we not meet with our anti-selves? The paper will explain it with the help of two-dimensional approach of the physical time that describe that the net temporal movement of the physical time is a constant zero.
\end{abstract}

Keyword: A Two-dimensional approach of the physical time, a unit vector of time, resultant macroscopic time, the positive and negative energies, local area of symmetry and parallel Universes.

\section{Introduction and Theory}

With regard of the concept of the parallel Universes [1], every space point has its equal and opposite anti-space point. Unlike the physical time [2], because the space point does not have any direction [3], the space point and its anti-space point are symmetric which means that the space point and anti-space point are the same point in space and they are separated by the fixed amount of physical time which is why the space point and anti-space point never meet with each other to make them both null and void. The space point and anti-space point are symmetric in space and asymmetric in time. The space point is the property of our Universe $[4,5]$ and anti-space point is the property of the anti-Universe in the Parallel Universe theory [6, 7]. The positive energies of our Universe [8] have anti-positive energies of the anti-
Universe and both are again separated by a fixed amount of time, the reason why, they do not annihilate each other. The same is true for negative energies of our Universe [9]; there are anti-negative energies of the anti-Universe and both are separated by a fixed amount of physical time frame. If there is a local area of symmetry in our Universe, there is the same amount of local area of symmetry in the Anti-Universe too because space is symmetric in the parallel Universes and the physical time is oppositely directed in the both the Universes. 


\section{International Journal of Science and Research (IJSR)

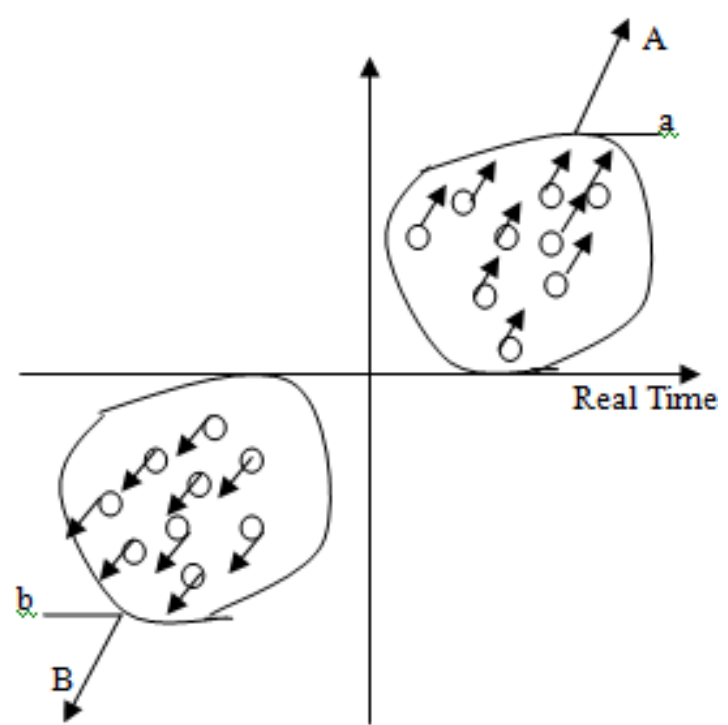

Figure 1: The area at the first quadrant is a local area of symmetry of ordinary matters in our Universe. The area in the third quadrant is the local area of symmetry of the antimatters in Anti-Universe. Both the local areas are space

symmetric but the resultant macroscopic times are oppositely directed (A \& B). a \& b are the real times ( $\mathrm{x}$-axis components of $\mathrm{A} \& \mathrm{~B})$.

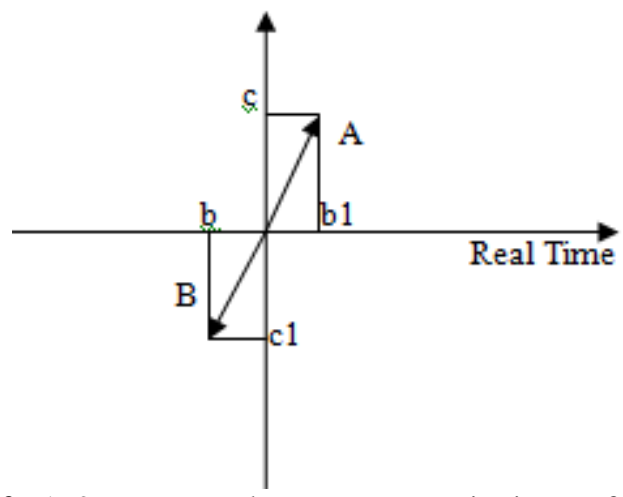

Figure 2: A \& B are resultant macroscopic times of the local areas of fig 1 . Both the areas are super imposing on each other in space but separated by real time $(b+b 1)$ and in

$$
\text { imaginary time }(c+c 1) \text {. }
$$

Because the physical times of the Universe and its antiuniverse are oppositely directed, they always yield a nonzero real time along x-axis $(b+b 1)$ and non-zero imaginary time along $y$-axis $(c+c 1)$. Because the real time and imaginary time are both non-zero, self and its anti-self will never meet each other because of the non-zero physical time differences in both real time and imaginary time as shown in the figure 2. The figure also describes the two dimensional approach of the physical time. Because the distance of $b$ and $b 1$ from the origin are the same, the net real time movement of the parallel Universes is a constant zero.

$$
\begin{gathered}
|b-0|=|0-b 1| \\
|b|=|-b 1|
\end{gathered}
$$

Similarly, the net imaginary time movement of the parallel Universes is also a constant zero.

$$
\begin{gathered}
|c-0|=|0-c 1| \\
|c|=|-c 1|
\end{gathered}
$$

If the local areas (of the figure 1) are extended to the parallel Universes, all the properties of the physical time are still hold the same $[10,11]$. Thus space and anti-space, energies and anti-energies, matters and anti-matters have identical identity in space but separated by the real times $(b+b 1)$ along $\mathrm{x}$-axis which human race can perceive and by the imaginary times $(c+c 1)$ along $\mathrm{y}$-axis which human race cannot perceive. Thus they will never meet with each other.

\section{On The Theory of Parallel Universes}

With regard of the concept of the parallel Universes [12, 13], every space point of our Universe has equal and opposite anti-space point in the anti-Universe which are identically placed in space and oppositely directed in time. Every amount of energy of our Universe (positive or negative) has equal and opposite anti-energy in the anti-Universe which are also identically placed in space but oppositely directed in time. Every matter of our Universe has equal and opposite anti-matter in the anti-Universe which are identically placed in space but oppositely directed in time. It is because time has a direction and a change of direction but space points does not have any direction. This is the reason why space is symmetric and the physical time is asymmetric for parallel Universes. Because times are mutually oppositely directed $[14,15]$ for the Universe and the anti-Universe [16, 17], an expanding Universe looks like a contracting anti-Universe. Thus Universe expansion and anti-Universe contraction are symmetric and flipped side of the same coin.

\section{Comparison of Real Time Components for Solid, Liquid and Gas of Both Parallel Universes}

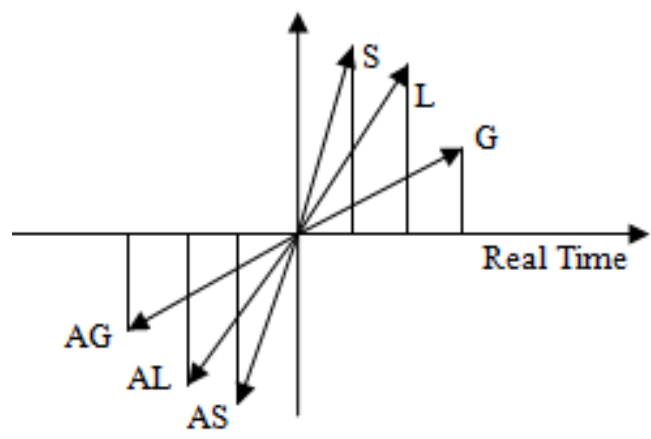

The first quadrant belongs to ordinary matters [18, 19] of different forms for our Universe [20, 21]; the solid (S) has less real time component, the liquid(L) has slightly higher real time component and the Gas has more real time component compared to solid and liquid. The imaginary time component is in reverse order as because all time vectors $\mathrm{S}, \mathrm{L}, \mathrm{G}$ have identical magnitudes and different directions. The third quadrant belongs to the Anti-Universe $[22,23]$ where same comparison can be seen for AG (antigas), AL (anti-liquid) and AS (anti-solid). The concept can be extended to energies $[24,25]$ and space points of both the Universe and the anti-Universe [26, 27]. Thus, time runs slower in solid compared to liquid and gas as real time 


\section{International Journal of Science and Research (IJSR) \\ ISSN (Online): 2319-7064 \\ Index Copernicus Value (2013): 6.14 | Impact Factor (2014): 5.611}

component is least for solid [28, 29]. As we understand only real part of time, for us, time runs slower in solid, little bit quicker in liquid and further quicker in gas. As we do not understand imaginary time, the imaginary part of time is psychologically disregarded.

\section{Conclusion}

A two-dimensional approach of the physical time introduces the concept of imaginary time. Only the real part of time can be psychologically recognizable. The imaginary part is psychologically disregarded as it is not recognizable. The real time runs slower in solid compared to liquid and gas. For our Universe, there is an equal and opposite antiUniverse; both are identically placed in space and oppositely directed in the physical time frame. Because the physical time is oppositely directed for both the Universes, an expanding Universe is equivalent to a contracting antiUniverse and vice versa. As the anti-Universe has equal and opposite amount of anti-matters, anti-energies and anti-space points compared to the matters, energies and space points of our Universe, thus time displacements also equal and opposite in nature which means for any self and anti-self, there are equal amount of real time displacement with respect to reference frame (the origin) and equal amount of imaginary time displacement with respect to the reference frame (the origin) which cancel out each other and the net result is that the net temporal movement of the parallel Universes is a constant zero. But because the real and imaginary time differences of self and anti-self is always non-zero, we can never meet with our anti-selves. This is a general truth for everything in the Universe and its antiUniverse.

\section{Acknowledgment}

I cordially admire Dr. Aparna Nath, Associate Professor and my $\mathrm{PhD}$ Guide, The department of Physics, National Institute of Technology, Agartala, India, for the epitome of inspiration and motivation to write this particular paper with perfection and accuracy. I am extremely thankful to her from all possible help she made to write this paper. Also I am thankful to The Department of Physics of National Institute Of Technology Agartala (NIT Agartala) for proper conduct and coordination.

\section{References}

[1] Prasenjit Debnath "The Space and the Physical Time Are Interdependent On Each-Other: The Numbers Associated With the Measurement of the Physical Time Are Macroscopic, Abstract and Deviation from Idealism" International Journal Of Science And Research, ISSN (online)- 2319-7064, volume 5 Issue 3, pp 276-279.

[2] Prasenjit Debnath, "Time and Space Are Mutually Inherent Property of Each Other, Where the Dependency of Time on Space Can Be Termed as Space-Time and the Dependency of Space on Time Can Be Termed as Time-Space" International Journal Of Science And Research, ISSN (online)- 2319-7064, volume 5 Issue 2, pp 1946-1948
[3] Brian Cox, Jeff Forshaw, "The quantum universe: everything that can happen does happen", Penguin Books, pp. 1-44.

[4] Stephen Hawking, "A Briefer History of Time”, Bantam Books, London, pp. 1-145.

[5] Stephen Hawking, "Black holes and Baby Universes and other essays", Bantam Press, London 2013, ISBN 978-0-553-40663-4

[6] Stephen Hawking, "The Grand Design”, Bantam Books, London 2011

[7] Stephen Hawking, "A Brief History of Time", Bantam Books, London 2011, pp. 156-157. ISBN-978-0-55310953-5

[8] Stephen Hawking, "The Universe in a Nutshell", Bantam Press, London 2013, pp. 58-61, 63, 82-85, 90 94, 99, 196. ISBN 0-553-80202-X

[9] Stephen Hawking, "The Beginning of Time", A Lecture.

[10] Stephen Hawking, “Stephen Hawking's Universe: Strange Stuff Explained", PBS site on imaginary time.

[11] Stephen Hawking, "How to build a time machine", 27 April, 2010.

[12] Uno Ingard, K "Fundamental of Waves \& oscillations", Cambridge University Press. P. 38, ISBN-0-521-33957XOxford: The British Academy, 1999

[13]A. Zee, "Quantum Field Theory in a Nutshell", Princeton University Press, 2003

[14] Storrs McCall, "A Model of the Universe", Oxford: Clarendon Press, 1994

[15]Craig Callender, "Time, Reality and Experience", Cambridge, UK: Cambridge University Press.

[16]Craig Callender, "Thermodynamic Asymmetry in Time", The Stanford Encyclopedia of Philosophy (Spring 2002 Edition)

[17] Storrs McCall, "A Model of the Universe", Oxford: Clarendon Press, 1994

[18]Robin Le Poidevin and Murray McBeath, "The Philosophy of Time" Oxford: Oxford University Press, 1993

[19] Newton-Smith, W.H., "The Structure of Time". London: Routledge \& Kegan Paul, 1980.

[20]Barry Dainton,'Time and Space", Ithaca: McGillQueen's University Press, 2001

[21] Robin Le Poidevin, "Questions of Time and Tense", Oxford: Oxford University Press, 1998.

[22] Nerhlich, Graham, "What Spacetime Explains". Cambridge: Cambridge University Press, 1994.

[23] Sklar, Lawrence, "Space, Time, and Space-time”. CA: University of California Press, 1974.

[24] Whitrow, G., "The Natural Philosophy of Time". Oxford: Oxford University Press, 1961. (2nd edn., 1980.)

[25]S.W. Hawking, and G.F.R. Ellis, "The Large Scale Structure of Space-Time", Cambridge University Press, (1973).

[26] Stephen Hawking, "A stubbornly persistent illusion-The essential scientific works of Albert Einstein”, Running Press Book Publishers, Philadelphia, London 2011.

[27] Flynn, John L, “Time travel literature”, on 29-09-2006 


\section{International Journal of Science and Research (IJSR) \\ ISSN (Online): 2319-7064}

Index Copernicus Value (2013): 6.14 | Impact Factor (2014): 5.611

[28] Stephen Hawking, "The Theory of Everything”, Jaico Books, pp. 1-110.

[29] Stephen Hawking, "The Illustrated A Brief History of Time”, Bantam Books, pp. 1-21.

\section{Author Profile}

Prasenjit Debnath, born in Agartala, Tripura, India on $15^{\text {th }}$ of March 1979. He is pursuing $\mathrm{PhD}$ degree in the Department of Physics in National Institute of Technology Agartala (NIT Agartala), India. 